\title{
Realization of $\mathcal{P} \mathcal{T}$-symmetric and $\mathcal{P} \mathcal{T}$-symmetry broken states in static optical lattice potentials
}

\author{
Felix Kogel, Sebastian Kotzur, Daniel Dizdarevic * Jörg Main, and Günter Wunner \\ Institut für Theoretische Physik 1, Universität Stuttgart, 70550 Stuttgart, Germany
}

(Dated: June 13, 2021)

\begin{abstract}
R. Labouvie et al. [Phys. Rev. Lett. 116, 235302 (2016)] have described an experiment with a weakly interacting Bose-Einstein condensate trapped in a one-dimensional optical lattice with localized loss created by a focused electron beam. We show that by setting suitable initial currents between neighboring sites it is possible to create $\mathcal{P} \mathcal{T}$-symmetric quasi-stationary and $\mathcal{P} \mathcal{T}$-symmetry broken decaying states in an embedded two-mode subsystem. This subsystem exhibits gain provided by the coupling to the reservoir sites and localized loss due to the electron beam, and shows the same dynamics as a non-Hermitian two-mode system with symmetric real and antisymmetric imaginary time-independent potentials, except for a proportionality factor in the chemical potential. We also show that there are three other equivalent scenarios, and that the presence of a localized loss term significantly reduces the size of the condensate required for the realization.
\end{abstract}

\section{INTRODUCTION}

In 1998 Bender and Boettcher [1] introduced a new class of Hamiltonians $\mathcal{H}$, which are invariant with respect to the combined effect of the parity operator $\mathcal{P}$ and the time-reversal operator $\mathcal{T}$, that is $[\mathcal{H}, \mathcal{P} \mathcal{T}]=0$, without being necessarily invariant with respect to either of them. This allows for non-Hermitian Hamiltonians with entirely real spectra within a so-called $\mathcal{P} \mathcal{T}$-symmetric regime, and otherwise an eigenvalue structure with complex conjugate pairs called $\mathcal{P} \mathcal{T}$-symmetry broken.

There is a wide range of applications for $\mathcal{P} \mathcal{T}$-symmetric systems, as non-Hermitian Hamiltonians are particularly suited to effectively describe open quantum systems (e.g. see [2]). They can, for example, be used to describe delocalization transitions in condensed matter [3], or for the investigation of population biology [4] and exceptional points $[5-8$. Furthermore, the concept of $\mathcal{P} \mathcal{T}$ symmetry can be applied to the fields of laser modes [9 11], electronic circuits 12 14, microwave cavities [15, or for the realization of unidirectional invisibility [16 18].

A direct observation of $\mathcal{P} \mathcal{T}$ symmetry is possible in optical systems [19, 20] due to the mathematical equivalence of the wave equation of electrodynamics in paraxial approximation and the Schrödinger equation. By considering light propagation in two wave guides the transition between the $\mathcal{P} \mathcal{T}$-symmetric and the $\mathcal{P} \mathcal{T}$-symmetry broken regime can be investigated. However, up to date an experimental observation of $\mathcal{P} \mathcal{T}$ symmetry in a quantum mechanical system is still lacking.

A promising experimental procedure for the realization of $\mathcal{P} \mathcal{T}$-symmetric quantum systems was proposed by considering a Bose-Einstein condensate in an optical double-well potential 21. As shown in Refs. 222 24. the formalism of $\mathcal{P} \mathcal{T}$ symmetry can be applied to such a nonlinear quantum system, which can develop stable $\mathcal{P} \mathcal{T}$-symmetric states [25]. In more recent works the

\footnotetext{
*daniel.dizdarevic@itp1.uni-stuttgart.de
}

use of bounded and unbounded states 26] or coupling to another Bose-Einstein condensate [27] were suggested to provide a coherent in- and out-coupling of particles. However, both methods are difficult to realize experimentally.

Kreibich et al. 28 30 proposed an experiment based on time-dependent optical lattices [31, in which the wells are loaded with Bose-Einstein condensates [32. In this way a two-mode system embedded into a larger multi-well system is created, which shows $\mathcal{P} \mathcal{T}$-symmetric dynamics in the mean-field approximation and beyond [33, 34. Although this approach effectively allows for the realization of $\mathcal{P} \mathcal{T}$ symmetry, the experimental setup is quite demanding and currently hardly realizable due to the time-dependent optical potentials.

In the present paper we will focus on an experiment with a time-independent optical lattice and localized Bose-Einstein condensates of ${ }^{87} \mathrm{Rb}$ atoms 35 . An electron beam can be used to create local losses at specific lattice sites [36]. In the following we will propose a modification of this experiment to realize $\mathcal{P} \mathcal{T}$-symmetric and $\mathcal{P} \mathcal{T}$-symmetry broken states in the mean-field approximation. It is sufficient to describe the system in the mean field, as effects beyond the mean-field theory do not play a role due to the large number of atoms present in the experiment.

\section{THEORY}

\section{A. Two-mode system}

To demonstrate the characteristics of $\mathcal{P} \mathcal{T}$ symmetry a two-well system filled with ultracold Bose-Einstein condensates is considered where the imaginary part of a complex potential describes the in- and out-coupling of particles. If the potential barrier separating the two wells is high enough, the respective wave functions can be assumed to be localized, so that the system is discrete. By using dimensionless units $(\hbar=m=1)$, the theoretical 
description of localized Bose-Einstein condensates in the $\mathcal{P} \mathcal{T}$-symmetric double well is given by the discrete GrossPitaevskii equation (e.g. see [37])

$$
\mathrm{i} \frac{\partial}{\partial t}\left(\begin{array}{l}
\psi_{1} \\
\psi_{2}
\end{array}\right)=\left(\begin{array}{cc}
g\left|\psi_{1}\right|^{2}+\mathrm{i} \gamma & -J \\
-J & g\left|\psi_{2}\right|^{2}-\mathrm{i} \gamma
\end{array}\right)\left(\begin{array}{l}
\psi_{1} \\
\psi_{2}
\end{array}\right),
$$

to which we will refer as the two-mode system (TMS) in the following. While the real part of the potential is symmetric, the imaginary part is anti-symmetric. The coherent coupling with the environment is given by the gain and loss factor $\gamma$, which effectively represents a complex potential. The factor $J$ is the coupling and describes the tunneling of the particles between the two wells. The Hamiltonian (1) represents a nonlinear system with the corresponding strength of nonlinearity $g=4 \pi a N$, where $N$ is the particle number and $a$ describes the scattering length according to the s-wave scattering in BoseEinstein condensates [38.

With the use of mean-field wave functions $\psi_{i}=$ $\sqrt{n_{i}} \exp \left(\mathrm{i} \varphi_{i}\right)$, where $n_{i}=\left|\psi_{i}\right|^{2}$ is the number of particles and $\varphi_{i}$ the phase of the condensate in the corresponding lattice site, the system in Eq. (1) can be solved. Under the assumption of a symmetric occupation distribution with $n_{i}=n_{0}$ the solution of the $\mathcal{P} \mathcal{T}$-symmetric system is given by

$$
\phi=\left(\begin{array}{c}
\sqrt{n_{0}} \exp (\mathrm{i} \varphi) \\
\sqrt{n_{0}} \exp (-\mathrm{i} \varphi)
\end{array}\right)
$$

with the phase

$$
\varphi=-\frac{1}{2} \arcsin \left(\frac{\gamma}{J}\right)
$$

The chemical potentials

$$
\mu=g n_{0} \pm \sqrt{J^{2}-\gamma^{2}}
$$

of these eigenstates are purely real for $|\gamma| \leq J$. In this case the time-evolved solutions $\psi_{i}(t)=\phi_{i} \exp (-\mathrm{i} \mu t)$ are stationary and constitute the so-called $\mathcal{P} \mathcal{T}$-symmetric solutions. For larger values of $\gamma$ the eigenvalues contain an imaginary part resulting in a time-dependent norm of the states, so that the $\mathcal{P} \mathcal{T}$ symmetry is broken.

In the following investigations our aim is to realize the stationary symmetric solutions of the system. Therefore, the accessible observables

$$
\begin{aligned}
& c_{k, l}=2 \sqrt{n_{k} n_{l}} \cos \left(\varphi_{l}-\varphi_{k}\right), \\
& j_{k, l}=2 J \sqrt{n_{k} n_{l}} \sin \left(\varphi_{l}-\varphi_{k}\right),
\end{aligned}
$$

i.e. the correlation $c$ and the net current $j$, are introduced in order to describe important properties of the dynamics of the $\mathcal{P} \mathcal{T}$-symmetric states. The corresponding characteristic values of the TMS

$$
\begin{aligned}
& c_{1,2}=2 n_{0} \sqrt{1-\left(\frac{\gamma}{J}\right)^{2}}, \\
& j_{1,2}=2 n_{0} \gamma,
\end{aligned}
$$

are time-independent and depend only on the phase difference of the two components of the wave function (2).

\section{B. Complex-extended wave functions}

Beyond the $\mathcal{P} \mathcal{T}$-symmetric regime with $|\gamma|>J$, the resulting complex eigenvalues (4) and time-dependent norm cause a time dependence of the Hamiltonian in Eq. (1). Nevertheless, for a time-independent Hamiltonian with a vanishing nonlinearity $g=0$, the wave functions (2) with a complex phase present solutions of the Schrödinger equation (1). By using the general relation

$$
\begin{aligned}
\arcsin (\alpha) & =-\mathrm{i} \ln \left(\mathrm{i} \alpha \pm \sqrt{1-\alpha^{2}}\right) \\
& =\frac{\pi}{2}-\mathrm{i} \ln \left(\alpha \pm \sqrt{\alpha^{2}-1}\right)
\end{aligned}
$$

for $|\alpha|>1$, the phase (3) turns into

$$
\varphi=-\frac{\pi}{4}+\frac{\mathrm{i}}{2} \ln \left(\alpha \pm \sqrt{\alpha^{2}-1}\right)
$$

and has the effect of shifting the initial occupations of each well so that the wave function reads

$$
\phi=\left(\begin{array}{l}
\sqrt{n_{0}\left(\alpha \pm \sqrt{\alpha^{2}-1}\right)} \mathrm{e}^{-\mathrm{i} \pi / 4} \\
\sqrt{n_{0}\left(\alpha \mp \sqrt{\alpha^{2}-1}\right)} \mathrm{e}^{\mathrm{i} \pi / 4}
\end{array}\right),
$$

where $\alpha=\gamma / J$. The two possible solutions in Eq. (9) correspond to the two solutions (4). For every such solution there are either exponentially increasing, or exponentially decreasing particle numbers

$$
n_{i}(t)=n_{i}(0) \mathrm{e}^{2 \operatorname{Im}(\mu) t},
$$

which correspond to the $\mathcal{P} \mathcal{T}$-symmetry broken states.

\section{Open few-mode model}

An experimental realization of the $\mathcal{P} \mathcal{T}$-symmetric and $\mathcal{P} \mathcal{T}$-symmetry broken states of the TMS requires a coherent in- and out-coupling of particles. It was shown that an out-coupling can be easily obtained with a focused electron beam [36]. An injection of particles can be realized by embedding the TMS into an optical lattice filled with Bose-Einstein condensates, which acts as a particle reservoir and allows for a steady current of particles into the system.

The mean-field description of a general open few-mode model is again given by the Gross-Pitaevskii equation,

$$
\begin{aligned}
\mathrm{i} \frac{\partial}{\partial t} \psi_{k}= & -J \psi_{k-1}-J \psi_{k+1}+g\left|\psi_{k}\right|^{2} \psi_{k} \\
& +\mu_{k} \psi_{k}-\mathrm{i} \frac{\gamma_{k}}{2} \psi_{k},
\end{aligned}
$$

where $\psi_{k}$ represents a Bose-Einstein condensate localized in the lattice site $k$ of a one-dimensional optical lattice 


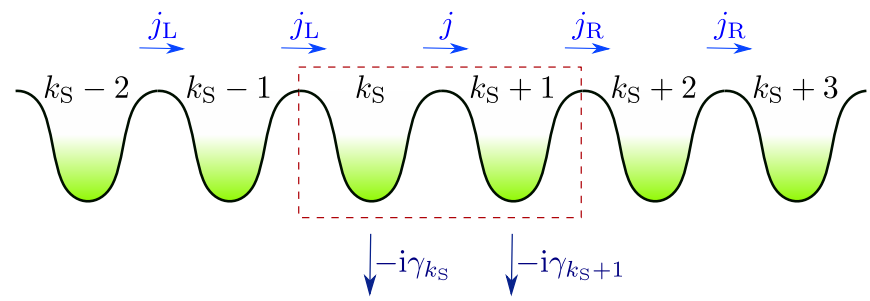

FIG. 1. The inner lattice sites with the currents $j_{\mathrm{L}}, j$ and $j_{\mathrm{R}}$ between them and two local loss terms $-\mathrm{i} \gamma_{k_{\mathrm{S}}}$ and $-\mathrm{i} \gamma_{k_{\mathrm{S}}+1}$ in the subsystem which is bounded by the dashed rectangle.

with the corresponding onsite energy $\mu_{k}$. The parameters $\gamma_{k}$ describe local in- or out-couplings of particles depending on their signs. The strength of the interaction $g$ is assumed to be equal to the strength of the nonlinearity $g$ of the $\mathcal{P} \mathcal{T}$-symmetric TMS at each lattice site. In order to ensure a simple realization, the tunneling coupling $J$ between neighboring lattice sites is assumed to be equal at all sites.

The differential equations (11) describe the experimental setup in [35]. In this experiment, roughly $45000{ }^{87} \mathrm{Rb}$ atoms were placed in a one-dimensional optical lattice, so that around 700 particles are located at each lattice site in the center of the potential. It was shown that the use of a strong loss term leads to the creation of a stationary state in the corresponding lattice site due to the quantum Zeno effect [39, 40]. However these stationary states do not have the characteristics of the solutions in the $\mathcal{P} \mathcal{T}$-symmetric TMS in equation (6). In the following, it is shown that the setting of appropriate initial phases and particle numbers of the condensates leads to stationary and exponentially decaying states with the $\mathcal{P} \mathcal{T}$ symmetric and $\mathcal{P} \mathcal{T}$-symmetry broken characteristics of the TMS, respectively.

\section{1. $\mathcal{P} \mathcal{T}$-symmetric regime}

In the following investigations the goal is to obtain the $\mathcal{P} \mathcal{T}$-symmetric states in the two sites of the subsystem labeled with $k_{\mathrm{S}}$ and $k_{\mathrm{S}}+1$. In particular the physically observable parameters, that is the particle number and the current, are desired to be realized as the constant characteristic values of the TMS. As the onsite energy leads to a shift in the energy of the system and has no influence on the dynamics it is set to zero. The characteristics of the subsystem have to fulfill the conditions of the TMS, viz.

$$
\begin{aligned}
n_{k_{\mathrm{S}}}=n_{k_{\mathrm{S}}+1} & \stackrel{!}{=} n_{0}, \\
j_{k_{\mathrm{S}}, k_{\mathrm{S}}+1} & \stackrel{!}{=} j=2 n_{0} \gamma .
\end{aligned}
$$

The other currents $j_{k, k+1}$ to the left of the subsystem are considered as equal and will be called $j_{\mathrm{L}}$. The same holds for the currents to the right, which will consequently be called $j_{\mathrm{R}}$. This special experimental setup
TABLE I. The different possibilities for the values of the currents $j_{\mathrm{L}}$ and $j_{\mathrm{R}}$ with the local gain and loss terms $\gamma_{k_{\mathrm{S}}}$ and $\gamma_{k_{\mathrm{S}}+1}$ assuming that the current $j$ in the subsystem is positive, $j>0$. Positive values of $\gamma_{k_{\mathrm{S}}}$ and $\gamma_{k_{\mathrm{S}}+1}$ correspond to the loss of particles, while negative values correspond to particle gain.

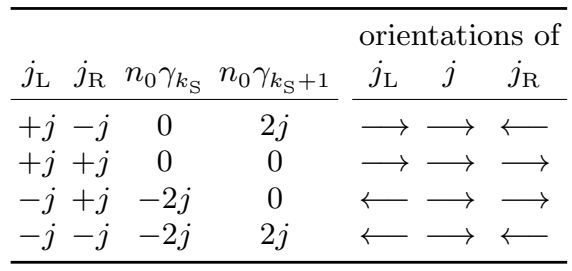

with constant localized loss terms $-\mathrm{i} \gamma_{k_{\mathrm{S}}}$ and $-\mathrm{i} \gamma_{k_{\mathrm{S}}+1}$, which only exist in the subsystem, is shown in Fig. 1. If these parameters $\gamma_{k}$ are negative, they act as a gain of particles in these lattice sites instead. In the $\mathcal{P} \mathcal{T}$ symmetric TMS the occupation in each well is a timeindependent constant, as there is the same amount of particles that are coupled in and out. For this reason it has to be ensured that the occupations are constant. Since

$$
\frac{\partial}{\partial t} n_{k}=j_{k-1, k}-j_{k, k+1}-\gamma_{k} n_{k},
$$

which follows from Eq. (11), the time-independence of the particle numbers of the subsystem yield the parameters

$$
\begin{aligned}
\gamma_{k_{\mathrm{S}}} & =\frac{j_{\mathrm{L}}-j}{n_{0}}, \\
\gamma_{k_{\mathrm{S}}+1} & =\frac{j-j_{\mathrm{R}}}{n_{0}} .
\end{aligned}
$$

To fulfill the requirements of time-independent currents and correlations in all lattice sites it can be shown by using Eq. (11) that all currents must have the same absolute values

$$
\left|j_{\mathrm{L}}\right| \stackrel{!}{=} j \stackrel{!}{=}\left|j_{\mathrm{R}}\right|
$$

assuming there is a positive current $j$ in the subsystem, i.e. from left to right (cf. Fig. 1). Moreover, all initial occupations have to be equally distributed throughout the lattice with $n_{k}(t=0)=n_{0}$ to produce the desired dynamics. As a result, there exist four different possibilities for the orientations of the currents $j_{\mathrm{L}}$ and $j_{\mathrm{R}}$ as shown in Table I. According to Eq. (5b), the derived initial values for the currents and particle numbers yield the phase differences

$$
\varphi_{k+1}-\varphi_{k}=\arcsin \left(\frac{j_{k, k+1}}{2 J \sqrt{n_{k} n_{k+1}}}\right),
$$

which have to be prepared initially. Consequently, all initial phases in Eq. (16) have to exhibit the same phase differences $\pm 2 \varphi$ of the TMS in Eq. (3) to ensure the stationary dynamics. 


\section{2. $\mathcal{P} \mathcal{T}$-symmetry broken regime}

In a manner similar to Sec. IIB we apply the approach of using complex phases to realize the $\mathcal{P} \mathcal{T}$-symmetry broken states of the TMS. It is expected that the characteristic dynamics for $\gamma>J$ can also be created by a preparation of the initial phases and occupations. To obtain analytic solutions in the following, the nonlinearity is set to zero, i.e. $g=0$. Since $g$ is freely adjustable by Feshbach resonances [41, this is no restriction with respect to experimental realizability.

The ansatz (16) with the current $j$ of the $\mathcal{P} \mathcal{T}$ symmetric states in Eq. $6 \mathrm{~b}$ yields the phase differences

$$
\varphi_{k+1}-\varphi_{k}= \begin{cases}\arcsin \left(s_{1} \frac{\gamma}{J}\right) & \text { for } k<k_{\mathrm{S}} \\ \arcsin \left(\frac{\gamma}{J}\right) & \text { for } k=k_{\mathrm{S}} \\ \arcsin \left(s_{2} \frac{\gamma}{J}\right) & \text { for } k>k_{\mathrm{S}}\end{cases}
$$

where the signs $s_{1}$ and $s_{2}$ can be selected independently, which corresponds to the four possibilities for the currents shown in Table I. Since $\gamma>J$, the initial complex phases have an impact on the initial occupations (see Eq. (7)), so that the time-dependent particle numbers of the subsystem follow as

$$
\begin{aligned}
n_{k_{\mathrm{S}}}(t) & =n_{0}\left(\alpha \pm \sqrt{\alpha^{2}-1}\right) \mathrm{e}^{2 \operatorname{Im}(\mu) t}, \\
n_{k_{\mathrm{S}}+1}(t) & =n_{0}\left(\alpha \mp \sqrt{\alpha^{2}-1}\right) \mathrm{e}^{2 \operatorname{Im}(\mu) t}
\end{aligned}
$$

with $\mu= \pm \sqrt{J^{2}-\gamma^{2}}$. If this is transferred to all lattice sites, all neighboring occupations obey the relation

$$
n_{k}(t)=n_{k+1}(t)\left(\alpha \pm \sqrt{\alpha^{2}-1}\right)^{2} .
$$

Thus, the effective initial preparation consists of exponentially distributed particle numbers and the same absolute phase differences of $\pi / 2$ between all lattice sites.

\section{RESULTS}

\section{A. Realizing $\mathcal{P} \mathcal{T}$ symmetry}

Using the results of Sec. [II a one-dimensional lattice with 50 sites is considered. The subsystem $\left\{k_{\mathrm{S}}, k_{\mathrm{S}}+1\right\}$ with $k_{\mathrm{S}}=25$ is located in the middle of this lattice with uniformly distributed optical characteristics. Without loss of generality we choose units such that $J=1$. Further, the nonlinearity $g$ is now set to zero both in the $\mathcal{P} \mathcal{T}$ symmetric and the $\mathcal{P} \mathcal{T}$-symmetry broken regimes. As the mean-field dynamics is independent of the particle number, the initial occupations can be set to $n_{k}(t=0)=0.5$. According to the four possibilities for the currents and
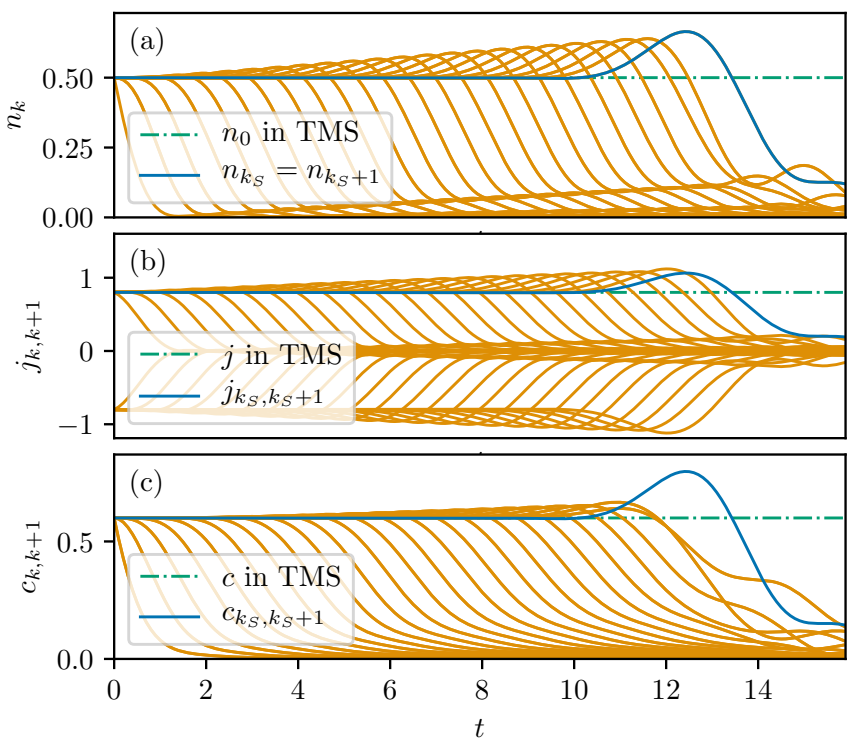

FIG. 2. The dynamics of the occupations, the currents, and the correlations of all 50 lattice sites for the initial currents $j_{\mathrm{L}}=j=-j_{\mathrm{R}}$ with the gain and loss term $\gamma=0.8$ and nonlinearity $g=0$. The occupations of the subsystem start to deviate significantly from the initial occupations at $t \approx 10$. The curves which decrease at a smaller time scale belong to the outer sites.

their respective gain and loss terms $\gamma_{k_{\mathrm{S}}}$ and $\gamma_{k_{\mathrm{S}}+1}$ in Table I. the phase differences must be equal to the values given in Eq. 16. Since the absolute phase shift is arbitrary, the phases of the subsystem are set to the values of the TMS given in Eq. (3), $\varphi_{k_{\mathrm{S}}}=-\varphi_{k_{\mathrm{S}}+1}=\varphi$.

To begin with, the first case for the currents in TableI is considered: The directions of all currents are aligned towards the subsystem in which, only in the right-hand site, a loss term with $\gamma_{k_{\mathrm{S}}+1}=4 \gamma$ exists. The resulting dynamics of the occupations and the correlations as well as the currents of all lattice sites is shown in Fig. 2 for the gain and loss factor $\gamma=0.8$.

It is noticeable that the occupation of the inner lattice sites in Fig. 22(a) remains approximately constant while the outer sites decrease one after another. Furthermore, all curves develop a local maximum. This is due to the fact that the tunneling currents exceed the initially set currents. The constant particle numbers of the subsystem remain at the constant value $n_{0}$ of the TMS until $t \approx 8$ when the adjoining sites can no longer maintain the appropriate current and thus the $\mathcal{P} \mathcal{T}$ symmetry breaks down.

The currents and correlations in the Figs. 2(b) and 2 (c) exhibit a similar behavior because they are directly correlated to the occupations. With the gain and loss factor that is used in Fig. 2 the characteristic values of the $\mathcal{P} \mathcal{T}$-symmetric regime are given by $c_{k_{\mathrm{S}}, k_{\mathrm{S}}+1}=0.6$ and $j_{k_{\mathrm{S}}, k_{\mathrm{S}}+1}=0.8$ according to Eqs. 6a) and 6b). These theoretical expectations of the TMS are depicted by the dash-dotted straight lines. 

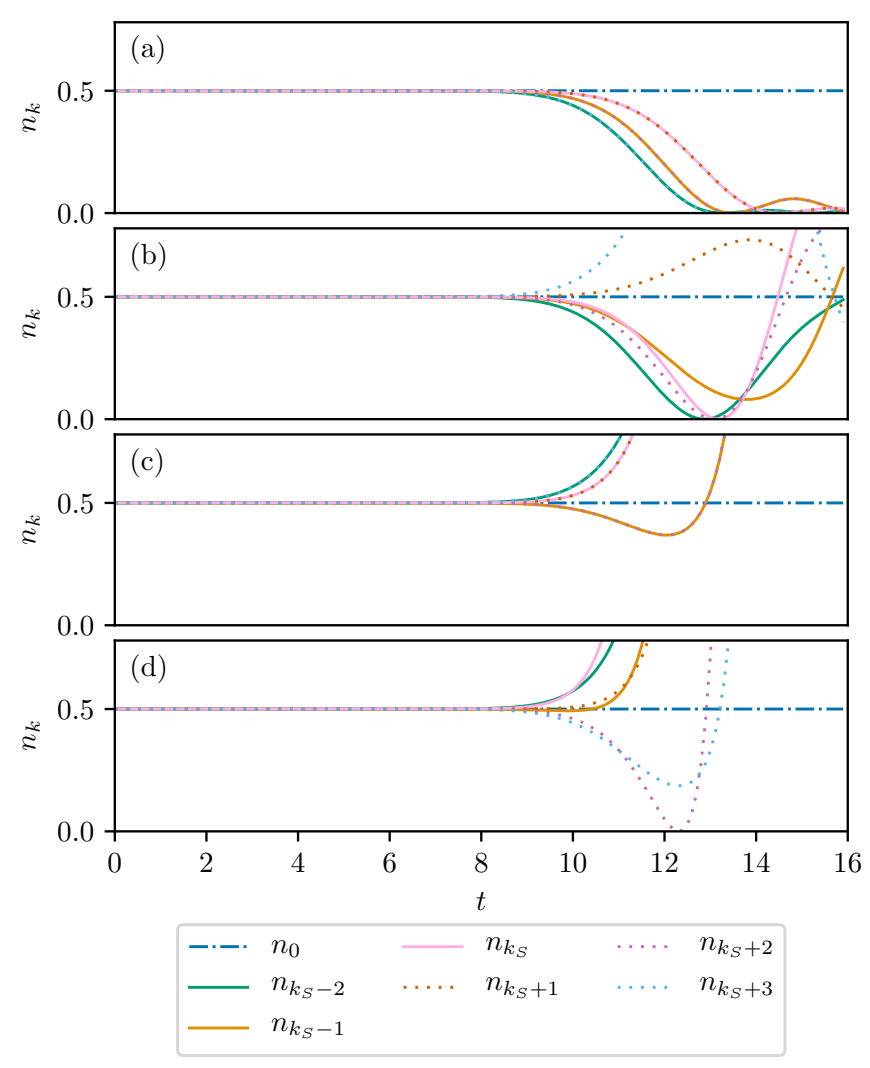

FIG. 3. The particle numbers $n_{k}(t)$ of the inner 6 lattice sites for $\gamma=1$ corresponding to the four possibilities of the currents $j_{\mathrm{R}}$ and $j_{\mathrm{L}}$ in Table [1] The nonlinearity is set to zero, $g=0$.

A comparison of the particle numbers $n_{k}(t)$ of the inner 6 lattice sites with $\gamma=1$ is illustrated in Fig. 3 for all four possibilities of the currents $j_{\mathrm{R}}$ and $j_{\mathrm{L}}$ in Table I] The local maxima in Fig. 3(a) do not appear here because the coupling constant $\gamma=1$ makes the tunneling current and the initial current equally large. For all four cases in Fig. 3 the occupations of the subsystem deviate from the constant value $n_{0}$ at $t \approx 8$, which is followed by chaotic dynamics. The second case in Fig. 3. (b) represents the trivial case with identical initial phases at all lattice sites and vanishing loss and gain terms because the currents of the reservoir sites effectively supply a balanced gain and loss term for the subsystem. In Figs. 3 (c) and 3(d) the particle numbers of the inner 6 lattice sites diverge after the quasi-stationarity breaks down at $t \approx 8$. As in Eq. (13) the gain term $\gamma_{k_{\mathrm{S}}}<0$ increases the occupation $n_{k_{\mathrm{S}}}$ exponentially. The gain and loss terms $\gamma_{k_{\mathrm{S}}}=-\gamma_{k_{\mathrm{S}}+1}$ in the fourth case look similar to those of the TMS in Eq. (1), but due to the interaction $J \neq 0$ with the adjoining sites, the dynamics of the subsystem finally collapses due to the finite reservoir. The time where the quasi-stationarity breaks down due to the emptying of the outer wells increases with the number of wells used as reservoirs. This time scale decreases for nonlinear interactions $g \neq 0$ as our investigations showed. However,

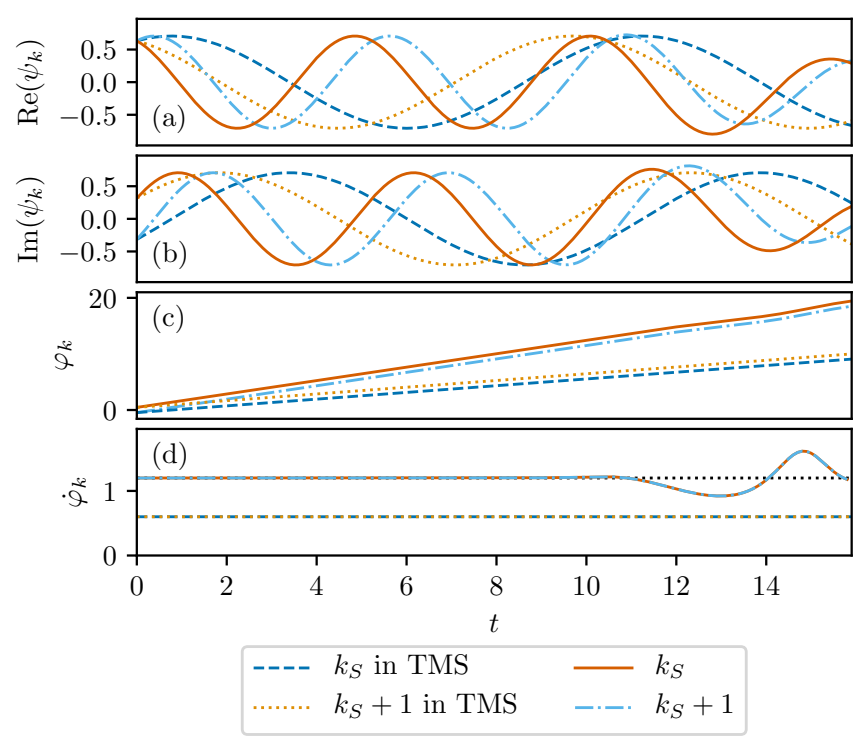

FIG. 4. The real and imaginary parts of the wave functions of the subsystem, as well as their phases and the derivatives of these phases are compared with the expected values of the TMS. The parameters are the same as in Fig. 2

since the nonlinearity does not lead to qualitative differences with respect to the realizability or the dynamics, we will focus on the linear case.

Due to the difficult realization of a gain term with $\gamma_{k_{\mathrm{S}}}<0$ in an actual experiment (e.g. see [4]), we consider the first two cases in Table [1] with the respective dynamics in Figs. 3(a) and 3(b) as experimentally accessible situations with $\mathcal{P} \mathcal{T}$-symmetric characteristics.

\section{Discussion of the phases}

Although the physical observables match the theoretically expected values, we will now consider the wave functions of the subsystem directly. To observe the oscillations of the phases it has to be ensured that the chemical potential does not vanish as for $\gamma=1$. Therefore, in the following we use $\gamma=0.8$.

The real and imaginary parts of these wave functions shown in Figs. 4(a) and 4(b) are qualitatively similar. They start at the correct values but evolve differently in time. Therefore, since the norms $\left|\psi_{k}(t)\right|^{2}=n_{k}(t)$ coincide with those of the TMS, the time-dependent phases $\varphi_{k}(t)$ of the wave functions $\psi_{k}(t)=\sqrt{n_{k}(t)} \exp \left(\mathrm{i} \varphi_{k}(t)\right)$, which are shown in Fig. 4 (c), have to be analyzed. While the phase differences of the subsystem and TMS are identical, their time derivatives $\dot{\varphi}_{k}(t)$ shown in Fig. 4 (d) are different.

Since the wave functions of the TMS evolve in time with the factor $\exp (-\mathrm{i} \mu t)$, the derivatives of their phases $\dot{\varphi}_{i}(t)=-\mu=0.6$ are half as large as those of the subsystem with the value $\dot{\varphi}_{k}(t<8)=1.2$. In Appendix A it is shown that the chemical potentials indeed differ from 


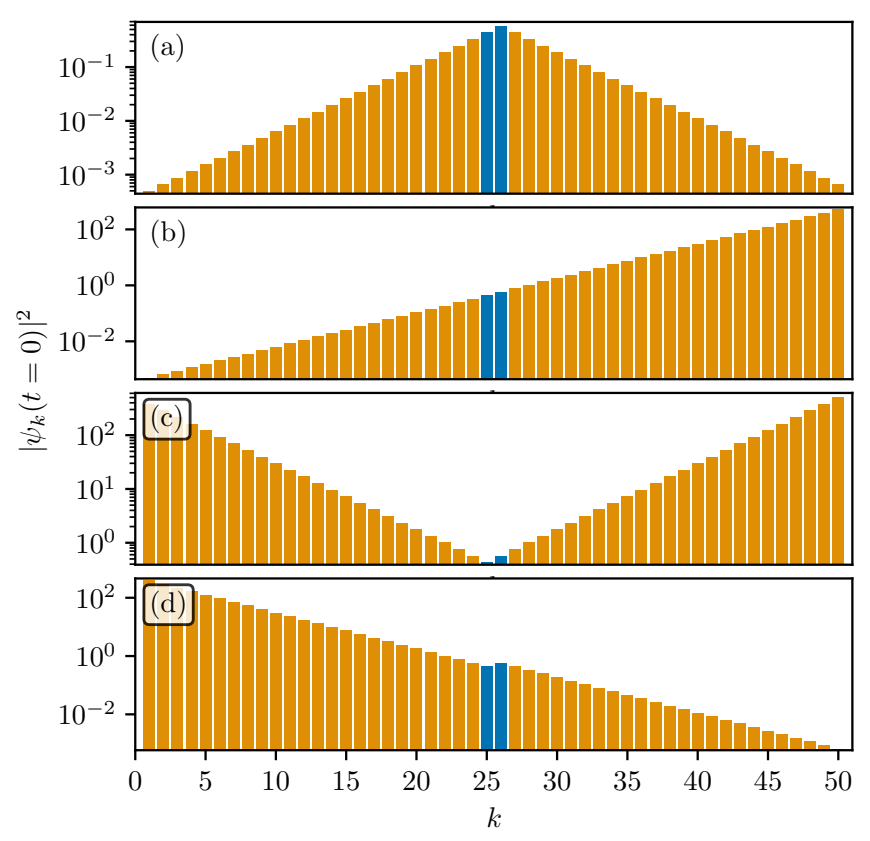

FIG. 5. The initial occupations $\left|\psi_{k}(t=0)\right|^{2}$ of the four possible decaying states with $\mu<0$ for the values of the currents from Table I with $\gamma=1.01$. The nonlinearity is set to zero, $g=0$.

one another by a factor of 2 .

For the general case of a non-vanishing nonlinearity $g \neq 0$ the wave functions of the subsystem have the form $\psi_{k}(t)=\psi_{k}(0) \exp (-\mathrm{i} \tilde{\mu} t)$ with the chemical potentials

$$
\tilde{\mu}=g n_{0} \pm 2 \sqrt{J^{2}-\gamma^{2}}
$$

whereas the wave functions of the TMS evolve in time with the chemical potentials (4). Since the currents and correlations in Eqs. (5a) and (5b) only depend on the phase differences, and the particle numbers are independent of any phases, the wave functions exhibit the same physical dynamics as those of the TMS. Due to no additional degrees of freedom in Sec. IIC1, the chemical potentials $\tilde{\mu}$ of the subsystem are not adjustable.

\section{B. Realizing broken $\mathcal{P} \mathcal{T}$ symmetry}

The aim of this subsection is to realize the $\mathcal{P} \mathcal{T}$ symmetry broken states of the TMS using the same setup as in the previous section. As derived in Sec. IIC2 the approach of complex phases leads to exponentially distributed initial occupations (see Eq. (19), which are illustrated in Fig. 5 for the four cases in Table II with $\gamma=1.01>J$. All four initial particle numbers of the subsystem are set to the same values given by Eq. (18) with $n_{0}=0.5$, where the sign belonging to the exponentially decaying occupations with $\mu<0$ is chosen. The nonlinearity is set to $g=0$ again, as a non-zero value disturbs the desired dynamics in Eq. (11) because of the
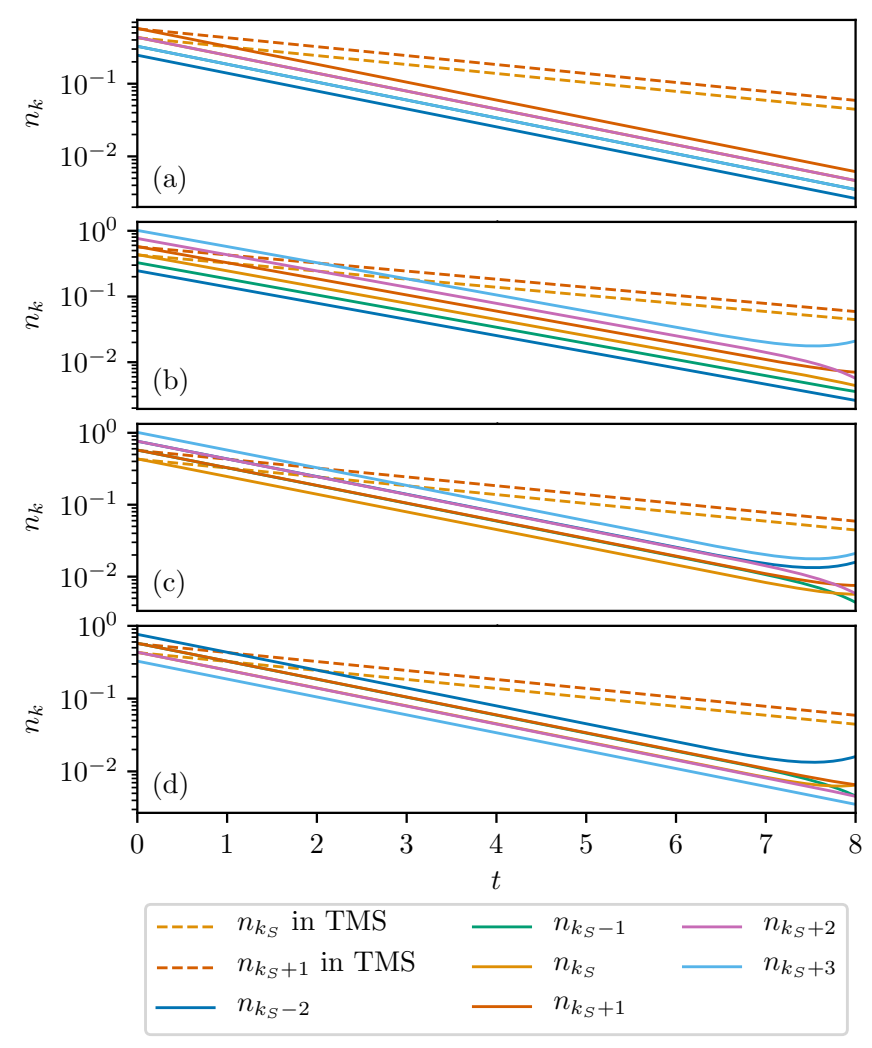

FIG. 6. The particle numbers $n_{k}(t)$ of the inner 6 lattice sites for $\gamma=1.01$ correspond to the four possible decaying states with the initial occupations shown in Fig. 5 The dash-dotted lines mark the expected particle numbers of the TMS.

time-dependent norm $\left|\psi_{k}\right|^{2}$. We want to emphasize that, although $n_{k_{\mathrm{S}}}$ and $n_{k_{\mathrm{S}}+1}$ are the same in every case shown in Fig. 5. the case with just localized loss (cf. Fig. 5(a)) requires only a surprisingly small overall number of particles in the condensate. Therefore, this approach seems particularly suitable for an experimental realization.

A comparison of the particle numbers $n_{k}(t)$ of the inner 6 lattice sites with $\gamma=1.01$ is illustrated in Fig. 6 for all four possibilities of the currents $j_{\mathrm{R}}$ and $j_{\mathrm{L}}$ in Table I It is noticeable that the occupations of the inner lattice sites evolve purely exponentially in time, but with a different factor compared to the TMS in Eq. 10. This stable behavior collapses roughly at the same time $t \approx 8$ as the $\mathcal{P} \mathcal{T}$-symmetric states in Fig. 3 .

A closer examination reveals that the exponentially decaying rates of these curves are identical in all subplots, and also twice as large as the ones of the TMS. As a consequence, the wave functions exhibit the chemical potential $\tilde{\mu}$ as the $\mathcal{P} \mathcal{T}$-symmetric wave functions in Eq. 20 with $g=0$. This complex eigenvalue results in time-dependent occupations

$$
n_{k}(t)=n_{k}(0) \mathrm{e}^{2 \operatorname{Im}(\tilde{\mu}) t}
$$

and is thus consistent with the wave functions of the $\mathcal{P} \mathcal{T}$ symmetric regime. 


\section{CONCLUSION}

In this paper we proposed an experimental setup for the realization of a two-mode quantum system consisting of Bose-Einstein condensates coupled to a reservoir, which leads to localized particle gain and loss. With a suitable choice of the particle numbers and phases, respectively, in each well the system exhibits either $\mathcal{P} \mathcal{T}$ symmetry or broken $\mathcal{P} \mathcal{T}$ symmetry for a finite time interval. In contrast to previous work, the theoretical treatment of the experiment is based on an open few-mode model with time-independent uniform optical characteristics.

All suitable initial phases allow for four types of environments, some with additional gain and loss in the inner wells, in which quasi-stationary states can be produced in a subsystem having almost the same dynamics as the $\mathcal{P} \mathcal{T}$-symmetric TMS. However, a closer inspection shows that the chemical potentials of both systems in the linear case are proportional by a factor of two. Thus, real and imaginary parts of the wave functions in the inner wells oscillate with different frequency. By continuing the phases of the $\mathcal{P} \mathcal{T}$-symmetric wave functions into the complex plane we find states with a time-dependent norm showing similar characteristics as the $\mathcal{P} \mathcal{T}$-symmetry broken solutions of the TMS. The resulting initial exponentially distributed particle numbers and equal absolute phase differences lead to wave functions with complex eigenvalues causing an exponential increase or decrease of the particle numbers in the inner wells, which differ from the behavior of the TMS again by the same factor of two.

For an actual experimental realization the situation with only localized loss seems particularly suited, as this approach can significantly reduce the number of particles required. Such localized losses can simply be created via a focused electron beam as shown in Ref. 35. The creation of arbitrary occupations in each lattice site is also experimentally possible [32, 43. However, the preparation of specific phase differences between Bose-Einstein condensates in neighboring sites, which is crucial for our approach, remains demanding. A possible experimental technique for such phase engineering may be to optically imprint the phases via far-off resonant lasers [44 47.

\section{Appendix A: Comparison of the chemical potential of the TMS and the subsystem in the lattice}

Here we show that the chemical potential of the subsystem in the lattice is twice as large as the one of the TMS assuming that the nonlinearity vanishes, $g=0$. The wave functions of the lattice site $k$

$$
\psi_{k}=\psi_{\mathrm{R}}+\mathrm{i} \psi_{\mathrm{I}}
$$

leaving out the indices due to clarity, is split into its real and imaginary parts. Thus the time derivative of the phase $\varphi=\arctan \left(\psi_{\mathrm{I}} / \psi_{\mathrm{R}}\right)$ can be calculated,

$$
\dot{\varphi}=\frac{1}{1+\left(\psi_{\mathrm{I}} / \psi_{\mathrm{R}}\right)^{2}} \frac{\dot{\psi}_{\mathrm{I}} \psi_{\mathrm{R}}-\psi_{\mathrm{I}} \dot{\psi_{\mathrm{R}}}}{\psi_{\mathrm{R}}^{2}}=\frac{1}{n}\left(\dot{\psi}_{\mathrm{I}} \psi_{\mathrm{R}}-\psi_{\mathrm{I}} \dot{\psi_{\mathrm{R}}}\right)
$$

with the particle number $\psi_{\mathrm{R}}^{2}+\psi_{\mathrm{I}}^{2}=n$. By using Eqs. 11 and (3), the derivatives of the phases of the TMS yield

$$
\begin{aligned}
\dot{\varphi}_{1}=\dot{\varphi}_{2} & =J \cos \left(\varphi_{2}-\varphi_{1}\right)=J \cos \left(\arcsin \left(\frac{\gamma}{J}\right)\right) \\
& =\sqrt{J^{2}-\gamma^{2}}=-\mu,
\end{aligned}
$$

which are equivalent to the expected value in Eq. (4) for $g=0$. In the same manner the derivatives of the phases at the initial time $t=0$, where all phase differences between neighboring sites have the same absolute value, can be calculated for a lattice described by

$$
\mathrm{i} \frac{\partial}{\partial t} \psi_{k}=-J \psi_{k-1}-J \psi_{k+1}-\mathrm{i} \frac{\gamma_{k}}{2} \psi_{k}
$$

Using the loss term $\gamma_{k_{S}+1}=4 \gamma$, for example, one finds

$$
\dot{\varphi}_{k_{\mathrm{S}}}=\dot{\varphi}_{k_{\mathrm{S}}+1}=2 J \cos \left(\varphi_{k_{\mathrm{S}}+1}-\varphi_{k_{\mathrm{S}}}\right)=-2 \mu=-\tilde{\mu},
$$

which explains the different behavior of the phases in the lattice system and in the TMS shown in Figs. 4(c) and $4(\mathrm{~d})$.
[1] C. M. Bender and S. Boettcher, Phys. Rev. Lett 80, 5243 (1998)

[2] E. M. Graefe, U. Günther, H. J. Korsch, and A. E. Niederle, J. Phys. A 41, 255206 (2008).

[3] N. Hatano and D. R. Nelson, Phys. Rev. Lett. 77, 570 (1996)

[4] D. R. Nelson and N. M. Shnerb, Phys. Rev. E 58, 1383 (1998).

[5] R. Gutöhrlein, H. Cartarius, J. Main, and G. Wunner, J. Phys. A 49, 485301 (2016)
[6] J. Schnabel, H. Cartarius, J. Main, G. Wunner, and W. D. Heiss, Acta Polytechnica 57, 454 (2017).

[7] L. Pan, S. Chen, and X. Cui, Phys. Rev. A 99, 011601(R) (2019)

[8] W. R. Sweeney, C. W. Hsu, S. Rotter, and A. D. Stone, Phys. Rev. Lett. 122, 093901 (2019)

[9] Y. D. Chong, L. Ge, and A. D. Stone, Phys. Rev. Lett. 106, $093902(2011)$

[10] L. Ge, Y. D. Chong, S. Rotter, H. E. Türeci, and A. D. Stone, Phys. Rev. A 84, 023820 (2011) 
[11] M. Liertzer, L. Ge, A. Cerjan, A. D. Stone, H. E. Türeci, and S. Rotter, Phys. Rev. Lett. 108, 173901 (2012)

[12] J. Schindler, Z. Lin, J. M. Lee, H. Ramezani, F. M. Ellis, and T. Kottos, J. Phys. A 45, 444029 (2012).

[13] J. Schindler, A. Li, M. C. Zheng, F. M. Ellis, and T. Kottos, Phys. Rev. A 84, 040101(R) (2011).

[14] H. Ramezani, J. Schindler, F. M. Ellis, U. Günther, and T. Kottos, Phys. Rev. A 85, 062122 (2012).

[15] S. Bittner, B. Dietz, U. Günther, H. L. Harney, M. MiskiOglu, A. Richter, and F. Schäfer, Phys. Rev. Lett. 108, 024101 (2012)

[16] Z. Lin, H. Ramezani, T. Eichelkraut, T. Kottos, H. Cao, and D. N. Christodoulides, Phys. Rev. Lett. 106, 213901 (2011)

[17] F. Loran and A. Mostafazadeh, Proc. R. Soc. A 472, $20160250(2016)$

[18] B. Lv, J. Fu, B. Wu, R. Li, Q. Zeng, X. Yin, Q. Wu, L. Gao, W. Chen, Z. Wang, Z. Liang, A. Li, and R. Ma, Sci. Rep. 7, 40575 (2017)

[19] A. Guo, G. J. Salamo, D. Duchesne, R. Morandotti, M. Volatier-Ravat, V. Aimez, G. A. Siviloglou, and D. N. Christodoulides, Phys. Rev. Lett. 103, 093902 (2009).

[20] C. E. Rüter, K. G. Makris, R. El-Ganainy, D. N. Christodoulides, M. Segev, and D. Kip, Nat. Phys. 6, 192 (2010)

[21] S. Klaiman, U. Günther, and N. Moiseyev, Phys. Rev. Lett. 101, 080402 (2008).

[22] H. Cartarius and G. Wunner, Phys. Rev. A 86, 013612 (2012)

[23] D. Dast, D. Haag, H. Cartarius, G. Wunner, R. Eichler, and J. Main, Fortschritte der Physik 61, 124 (2013).

[24] D. Dast, D. Haag, H. Cartarius, J. Main, and G. Wunner, J. Phys. A 46, 375301 (2013).

[25] D. Haag, D. Dast, A. Löhle, H. Cartarius, J. Main, and G. Wunner, Phys. Rev. A 89, 023601 (2014).

[26] F. Single, H. Cartarius, G. Wunner, and J. Main, Phys. Rev. A 90, 042123 (2014).

[27] R. Gutöhrlein, J. Schnabel, I. Iskandarov, H. Cartarius, J. Main, and G. Wunner, J. Phys. A 48, 335302 (2015)

[28] M. Kreibich, J. Main, H. Cartarius, and G. Wunner, Phys. Rev. A 87, 051601(R) (2013).

[29] M. Kreibich, J. Main, H. Cartarius, and G. Wunner, Phys. Rev. A 90, 033630 (2014).

[30] M. Kreibich, J. Main, H. Cartarius, and G. Wunner, Phys. Rev. A 93, 023624 (2016).
[31] K. Henderson, C. Ryu, C. MacCormick, and M. G. Boshier, New J. Phys. 11, 043030 (2009).

[32] S. Peil, J. V. Porto, B. Laburthe Tolra, J. M. Obrecht, B. E. King, M. Subbotin, S. L. Rolston, and W. D. Phillips, Phys. Rev. A 67, 051603(R) (2003)

[33] D. Dizdarevic, J. Main, K. Alpin, J. Reiff, D. Dast, H. Cartarius, and G. Wunner, Phys. Rev. A 97, 013623 (2018)

[34] T. Mathea, D. Dast, D. Dizdarevic, H. Cartarius, J. Main, and G. Wunner, J. Phys. A 51, 315303 (2018)

[35] R. Labouvie, B. Santra, S. Heun, and H. Ott, Phys. Rev. Lett. 116, 235302 (2016)

[36] G. Barontini, R. Labouvie, F. Stubenrauch, A. Vogler, V. Guarrera, and H. Ott, Phys. Rev. Lett. 110, 035302 (2013).

[37] E.-M. Graefe, J. Phys. A 45, 444015 (2012).

[38] C. J. Pethick and H. Smith, Bose-Einstein Condensation in Dilute Gases, 2nd ed. (Cambridge University Press, 2008).

[39] B. Misra and E. C. G. Sudarshan, J. Math. Phys. 18, 756 (1977)

[40] A. G. Kofman and G. Kurizki, Nature 405, 546 (2000),

[41] S. Inouye, M. R. Andrews, J. Stenger, H.-J. Miesner, D. M. Stamper-Kurn, and W. Ketterle, Nature 392, 151 (1998)

[42] N. P. Robins, C. Figl, M. Jeppesen, G. R. Dennis, and J. D. Close, Nature Physics 4, 731 (2008)

[43] P. Würtz, T. Langen, T. Gericke, A. Koglbauer, and H. Ott, Phys. Rev. Lett., 103, 080404 (2009).

[44] L. Dobrek, M. Gajda, M. Lewenstein, K. Sengstock, G. Birkl, and W. Ertmer, Phys. Rev. A 60, R3381 (1999)

[45] J. Denschlag, J. E. Simsarian, D. L. Feder, C. W. Clark, L. A. Collins, J. Cubizolles, L. Deng, E. W. Hagley, K. Helmerson, W. P. Reinhardt, S. L. Rolston, B. I. Schneider, and W. D. Phillips, Science 287, 97 (2000).

[46] S. Martellucci, A. Chester, A. Aspect, and M. Inguscio, Bose-Einstein Condensates and Atom Lasers (Springer US, 2007).

[47] S.-W. Song, D.-S. Wang, H. Wang, and W. M. Liu, Phys. Rev. A 85, 063617 (2012). 